\title{
Wilson Disease
}

National Institute of Neurological Disorders and Stroke (NINDS)

\section{Source}

National Institute of Neurological Disorders and Stroke (NINDS). Wilson Disease

Information Page.

Wilson disease (WD) is a rare inherited disorder of copper metabolism in which excessive amounts of copper accumulate in the body. The buildup of copper leads to damage in the liver, brain, and eyes. Although copper accumulation begins at birth, symptoms of the disorder only appear later in life. The most characteristic sign of WD is the KayserFleisher ring - a rusty brown ring around the cornea of the eye that can best be viewed using an ophthalmologist's slit lamp. The primary consequence for most individuals with WD is liver disease, appearing in late childhood or early adolescence as acute hepatitis, liver failure, or progressive chronic liver disease in the form of chronic active hepatitis or cirrhosis of the liver. In others, the first symptoms are neurological, occur later in adulthood, and commonly include slurred speech (dysarthria), difficulty swallowing (dysphagia), and drooling. Other symptoms may include tremor of the head, arms, or legs; impaired muscle tone, and sustained muscle contractions that produce abnormal postures, twisting, and repetitive movements (dystonia); and slowness of movements (bradykinesia). Individuals may also experience clumsiness (ataxia) and loss of fine motor skills. One-third of individuals with WD will also experience psychiatric symptoms such as an abrupt personality change, bizarre and inappropriate behavior, depression accompanied by suicidal thoughts, neurosis, or psychosis. WD is diagnosed with tests that measure the amount of copper in the blood, urine, and liver. 